\title{
Littoralisation et ségrégation socio-spatiale : le cas du quartier Balmer de San-Pédro (Côte d'Ivoire)
}

\section{Florent Gohourou, Cédric Audebert et Émile Aurélien Ahua}

\section{(2) OpenEdition}

Édition électronique

URL : http://journals.openedition.org/belgeo/37207

DOI : $10.4000 /$ belgeo.37207

ISSN : 2294-9135

Éditeur :

National Committee of Geography of Belgium, Société Royale Belge de Géographie

Référence électronique

Florent Gohourou, Cédric Audebert et Émile Aurélien Ahua, « Littoralisation et ségrégation sociospatiale : le cas du quartier Balmer de San-Pédro (Côte d'Ivoire) », Belgeo [En ligne], 1 | 2020, mis en ligne le 10 avril 2020, consulté le 20 janvier 2021. URL : http://journals.openedition.org/belgeo/37207 ; DOI : https://doi.org/10.4000/belgeo.37207

Ce document a été généré automatiquement le 20 janvier 2021.

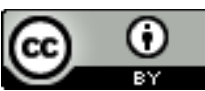

Belgeo est mis à disposition selon les termes de la licence Creative Commons Attribution 4.0 International. 


\title{
Littoralisation et ségrégation socio- spatiale : le cas du quartier Balmer de San-Pédro (Côte d'Ivoire)
}

\author{
Florent Gohourou, Cédric Audebert et Émile Aurélien Ahua
}

\section{Introduction}

1 Zones de contact entre terre et océan, les littoraux constituent des milieux riches et attractifs pour un large éventail d'activités, compte tenu de la diversité de leurs biotopes marins et côtiers ainsi que de la multiplicité des possibilités économiques offertes par leur environnement. Cela a pour corollaire une forte anthropisation, parmi les plus poussées au monde (Seck, 2014). L'attraction des milieux littoraux et côtiers s'est traduite par une forte littoralisation de ces espaces en lien avec une croissance démographique exponentielle. Les littoraux sont alors devenus le théâtre d'une multiplicité de pressions anthropiques (Robert, 2008), usages et activités parfois complémentaires, parfois opposés, souvent réinventés, au gré des rapports tissés par les individus et les collectifs avec la mer (Desse, 2005, Guyot, 2009, Cadoret, 2006), avec des enjeux légaux de plus en plus importants (Pognonec, 2016).

2 Au cours des dernières décennies, ces espaces sont devenus des sites d'installation permanente car ils ont attiré à la fois des populations riches et/ou pauvres. Ainsi, l'évolution des franges littorales a été marquée par divers modes d'artificialisation des côtes, qui se sont accompagnés de mutations et bouleversements socioéconomiques et culturels tout aussi variés que traduisent notamment les dynamiques de ségrégation.

Les pratiques ségrégationnistes aux dimensions tant sociales, psychologiques que spatiales représentent une problématique d'intérêt pour le géographe (Bignoumba, 2000), qui par le jeu des échelles est en mesure de porter un regard éclairant sur la question. Les dimensions sociale et ethnique constituent, entre autres variables, des éléments explicatifs parmi les plus importants dans ce processus dans les grandes villes européennes (Audren et Baby-Collin, 2017, Prétéceille, 2009) comme en Côte d'Ivoire 
(Gohourou, 2012). A San-Pédro, ville côtière du Sud-Ouest de la Côte d'Ivoire, l'espace littoral du quartier Balmer attire les hommes en raison de la qualité paysagère de son environnement maritime et de ses sites balnéaires et du dynamisme des activités économiques qui y sont développées.

De fait, la mise en valeur du littoral de Balmer est marquée par un double processus. D'une part, une population d'origine étrangère met en valeur l'espace dans le cadre d'une économie résidentielle et touristique. D'autre part, une population locale, plutôt pauvre, développe d'autres stratégies de mise en valeur en développant une palette d'activités commerciales qui accompagnent l'essor démographique. Dans le même temps, on constate que l'accès à la plage et aux différents espaces balnéaires fait l'objet d'une ségrégation et de difficultés de cohabitation entre les acteurs qui fréquentent les lieux concernés. Dès lors, notre étude vise à comprendre les facteurs explicatifs qui sous-tendent l'émergence du phénomène de ségrégation littorale à Balmer. Après avoir présenté le matériel et la méthode, notre réflexion s'attachera à montrer dans un premier temps les modes de littoralisation dans la zone d'étude. Ensuite, il sera question d'identifier les acteurs en présence. Enfin, nous analyserons les effets de la ségrégation à l'œuvre dans la zone.

\section{Matériel et méthodes de travail}

Le présent article s'inscrit dans la lignée des études qui postulent que la littoralisation et l'urbanisation littorale influent de manière directe sur l'émergence des différenciations socio-spatiales et la reproduction des systèmes ségrégationnistes. L'analyse du phénomène de ségrégation sur le littoral nécessite l'adoption d'une méthode quasi-complexe et adaptative. Ainsi, la démarche utilisée dans cette étude s'est construite autour de la triade Espace, Temps, Identité des acteurs, et s'appuie sur trois temps distincts susceptibles de révéler des usages spécifiques de l'espace: le matin (8h-12h), l'après-midi (14h-18h) et la soirée (18h-21h).

6 L'aménagement du territoire et l'organisation socio-spatiale des différents acteurs sur le littoral constituant des dimensions fondamentales du phénomène ségrégatif, l'analyse des flux migratoires des populations et la structuration des réseaux d'acteurs nous ont conduit à recourir à une analyse mixte, à la fois qualitative et quantitative, alimentée par une approche documentaire sur les questions de littoral et de ségrégation.

7 La divergence d'intérêts et de perceptions dans la mise en valeur et la territorialisation du littoral est étroitement liée aux rythmes de la ségrégation entre les populations pratiquant ce milieu. A ce titre, un questionnaire a été adressé aux différents acteurs qui fréquentent et s'approprient l'espace littoral de Balmer. Ce questionnaire avait pour objectif d'analyser les rapports entre les différents acteurs en présence. Les variables Temps/Espace étant des éléments de combinaison et/ou de discontinuité concernant les activités observées et la mise en valeur de ce territoire, les interrogations soumises aux différentes populations littorales consistaient à identifier les motivations de l'attractivité du littoral de Balmer auprès des populations (pourquoi ?), les différents usages côtiers et "valeurs" attribuées au littoral (comment?), les temporalités variées et le contexte de fréquentation de cet espace (où, quand, quelle heure ?) et enfin le profil et l'identité des acteurs (origine sociale ? statut économique?). 
Une autre séquence d'analyse nous est apparue riche d'enseignements et pertinente pour comprendre les logiques observées. Il s'agit de la méthode du parcours commenté accompagné d'une observation de terrain, qui offre un échange et un dialogue pénétrant avec un acteur du quotidien qui est en même temps un témoin de l'évolution $\mathrm{du}$ territoire. Cette méthode nous a permis d'identifier les différents acteurs en présence, les différents espaces fréquentés, prisés et discrédités. En outre, elle nous a permis d'appréhender les déterminants de la ségrégation littorale à Balmer, de saisir l'organisation des différents réseaux (stations balnéaires, espaces crées et fréquentés), ainsi que les groupes sociaux (riches ou pauvres), leur univers culturel (oralité, type de loisirs, nourriture, tenues vestimentaires, etc.), et les fluidités socio-spatiales des individus et des collectifs.

9 Au total, ce sont 194 acteurs du littoral qui ont été enquêtés par questionnaire, parmi lesquels 50 résidents, 21 touristes et 123 actifs dans les secteurs formels et informels qui ont constitué l'échantillon de l'étude. Quant à l'enquête par entretien, elle a consisté à interviewer les autorités locales (Mairie, Direction des affaires maritimes, autorités portuaires) afin de savoir comment ces gestionnaires du littoral appréhendent les différentiations sociales existantes et la façon dont ils ambitionnent de pallier à ces inégalités. Les données obtenues sur le terrain ont fait l'objet d'un traitement numérique et cartographique à l'aide des logiciels ArcMap 10.4 et Excel. Enfin, des données statistiques de l'Institut National de la Statistique (INS) et l'exploitation de Google Earth ont également aidé à la réalisation de notre objectif.

Figure 1. Localisation de l'espace d'étude.

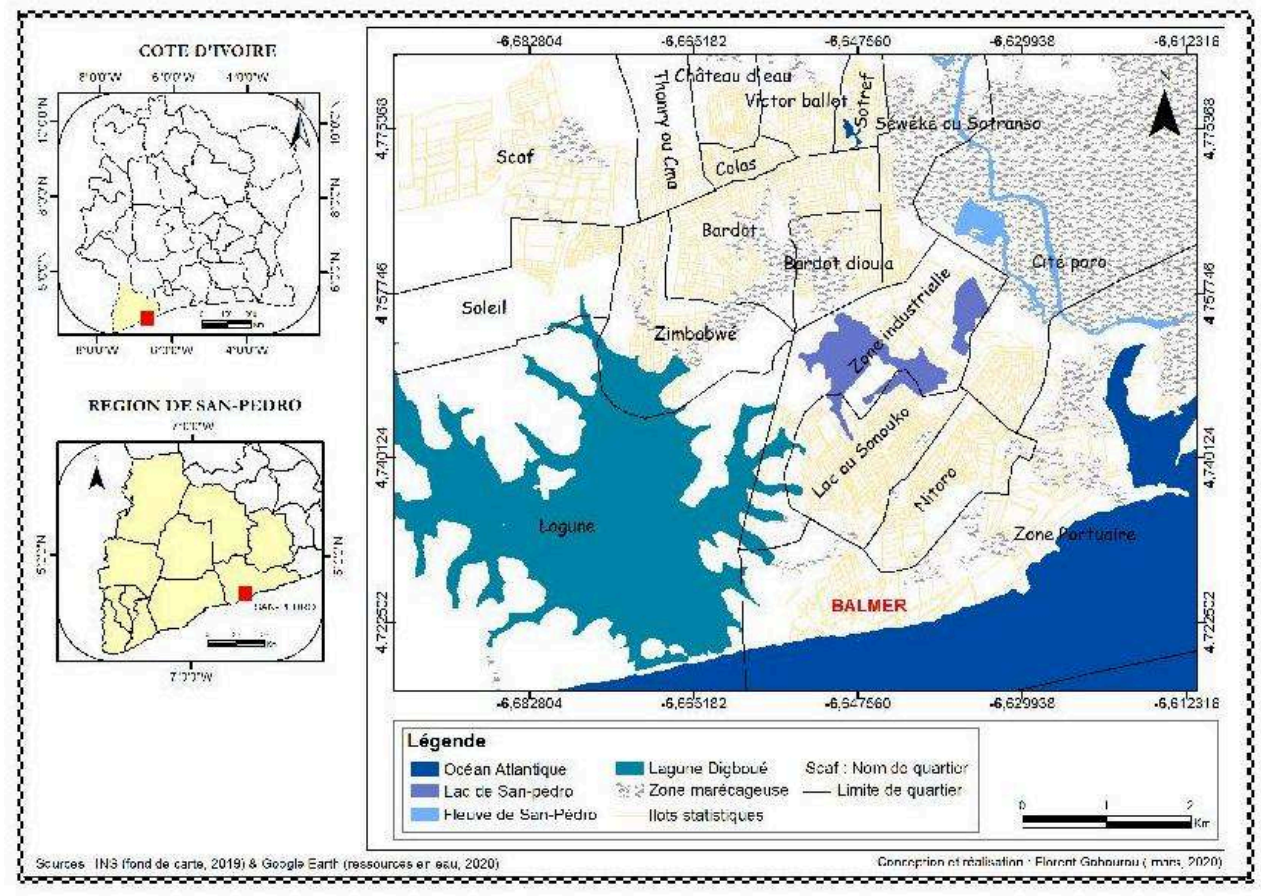




\section{Résultats : segmentation spatiale et spatialisation des inégalités}

\section{Littoralisation, urbanisation et ségrégation spatiale}

Dans le District du Bas Sassandra qui comprend les régions de la Nawa, du Gbôklê et de San-Pédro, la poussée démographique et urbaine est une réalité quotidienne puisque selon l'INS $(1988,1998,2014)$, le taux d'urbanisation y est passé de 14,2 \% en 1988, à 12,3 \% en 1998, puis à 27,6 \% en 2014 ; soit un taux d'urbanisation global de 19,3\%.

11 Entre 1988 et 2014, en passant de 31606 à 209590 habitants (INS, 1988, 2014), la ville de San-Pédro a connu une croissance démographique exponentielle qui s'est accompagnée d'un étalement rapide de l'espace urbain. Avec un effectif total de 10961 habitants en 1988, de 18821 habitants en 1998 et de 37338 habitants en 2014 (INS, 1988, 1998, 2014), les espaces littoraux de San-Pédro sont devenus de fait les zones prioritairement ciblées par les mouvements de population et les itinéraires privilégiés des populations riveraines, qui se déploient en grand nombre de manière temporaire ou permanente. Ainsi, la littoralisation et l'urbanisation littorale ont atteint un niveau sans précédent, dont les effets s'incarnent dans la co-présence de populations aux caractéristiques et pratiques variées sur le littoral, notamment à Balmer.

\section{L'attractivité du quartier Balmer : des phases successives de littoralisation}

12 L'urbanisation littorale à San-Pédro a connu deux phases : la première correspondant à l'essor de l'économie portuaire et halieutique autour des années 1980 et la seconde, en cours actuellement, est relative à la naissance de l'économie touristique, à la prolifération de petits commerces, et au développement résidentiel. La première phase de cette littoralisation a été induite par les populations étrangères (Libanais, Français), principales actrices du secteur commercial et de l'économie industrielle locale. Cette phase a été marquée par l'implantation de logements de haut standing près de la mer et la création d'un quartier de type résidentiel.

Figure 2. Constructions de haut standing au quartier Balmer.
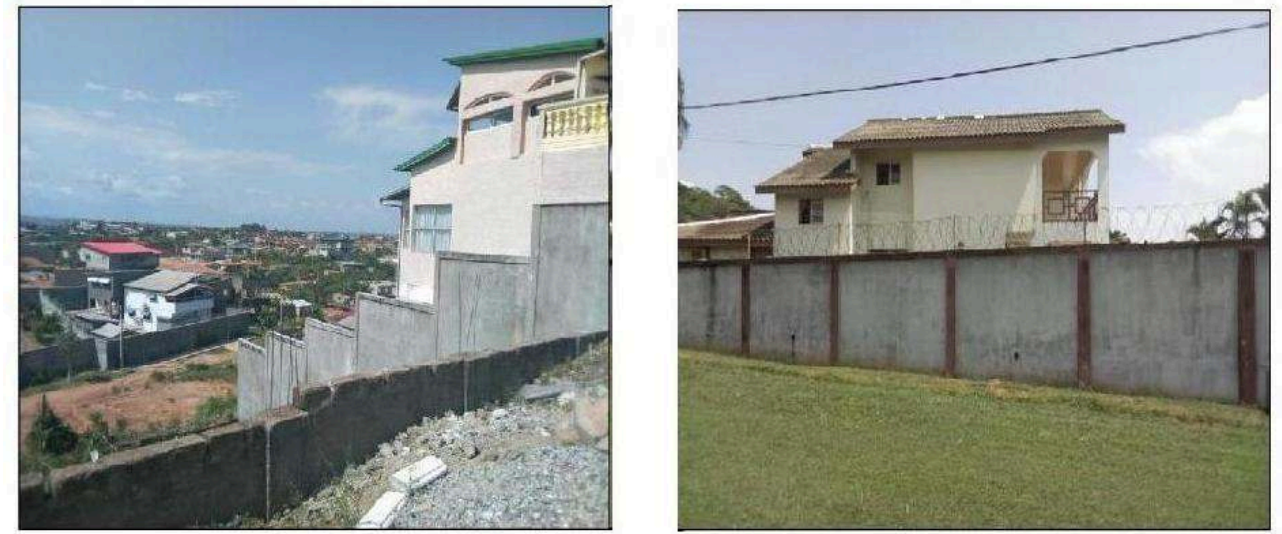

Source : E. A. Ahua, janvier 2019

13 L'aspect caractéristique que présentent ces édifices, le statut des populations qui y résident et les perceptions sociales auxquelles elles renvoient ont conféré au quartier 
Balmer le statut d'espace résidentiel et de luxe dans la ville. Avant le démarrage de la seconde phase de littoralisation, une politique touristique a été mise en place localement en réponse à l'arrivée croissante de touristes étrangers attirés par les belles plages, les côtes rocheuses, les berges renommées et une culture halieutique ivoirienne qui suscitait la curiosité. Cette dynamique touristique a eu pour conséquence la construction d'hôtels, de restaurants et autres lieux gastronomiques, de loisirs (piscines, espaces verts, musées, etc.) et de villégiature adaptés à la nouvelle clientèle (fig. 3). La première phase de littoralisation marquée par un essor à la fois résidentiel et touristique s'est accompagnée de l'installation d'une population uniquement étrangère.

Figure 3. Espaces gastronomiques et de loisirs sur le littoral de Balmer.
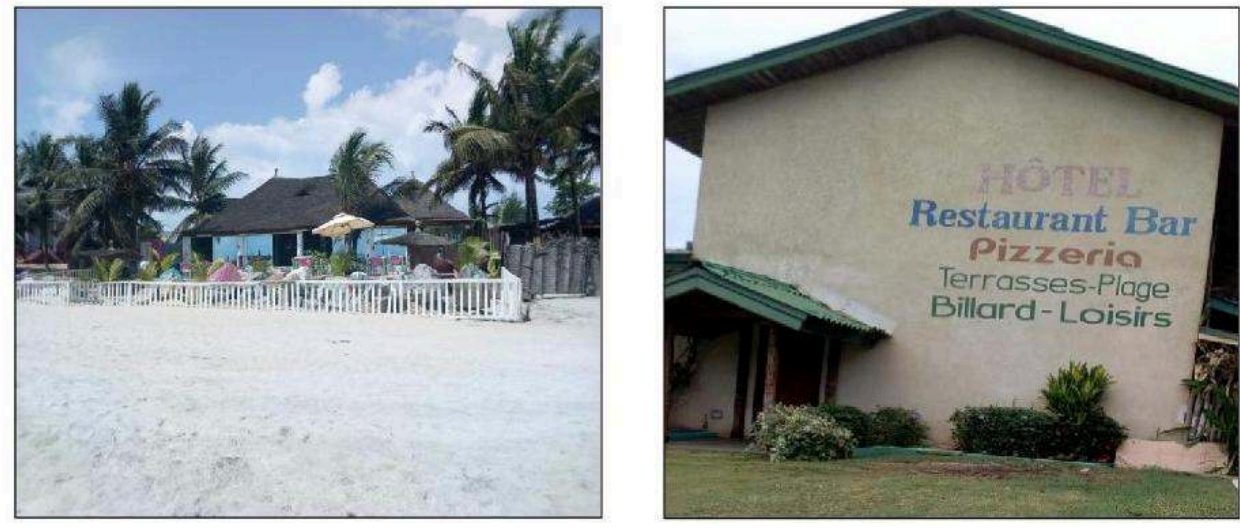

Source : E. A. Ahua, janvier 2019

La seconde phase de littoralisation s'est développée en lien avec l'attractivité de la plage et le développement des activités balnéaires. L'un des points de différenciation de ce type de littoralisation par rapport à la première phase réside dans la temporalité ponctuelle, temporaire - des usages que développent les acteurs. Il s'agit principalement de populations locales, dont les pratiques se caractérisent par des flux ponctuels de visites guidées, de séances de natation et autres activités de détente. Ce type de littoralisation est le fait de mobilités récurrentes et limitées dans le temps de populations ivoiriennes, en général les week-ends (samedis et dimanches). Elles constituent une clientèle potentielle pour d'autres acteurs qui développent de petits commerces dans la vente d'aliments, de produits pharmaceutiques et cosmétiques, etc. Le dynamisme de ces activités a suscité à son tour une vocation plus résidentielle et économique de ces espaces de la part des populations. La seconde phase de la littoralisation prend donc forme avec l'installation de populations moins nanties à Balmer, et le développement attenant de l'habitat précaire. Elle se traduit donc par la diversification du tissu social, de l'origine des habitants et de la nature du bâti résidentiel dans le quartier.

\section{Des territorialisations distinctes du littoral par les acteurs en présence : combinaison ou opposition d'activités économiques et de loisirs ?}

Les différents acteurs présents sur le littoral de Balmer se sont installés pour divers motifs. D'une part, les populations étrangères à haut revenu sont à la recherche d'un cadre résidentiel favorable leur offrant bien-être, repos, détente et loisirs. D'autre part, des populations à faibles revenus en quête d'une vie meilleure sont motivées par une 
représentation favorable de la localité, qu'ils identifient à un espace d'opportunités pour l'essor d'une activité économique (halieutique ou informelle).

Par ailleurs, malgré la prépondérance de l'habitat anarchique et précaire, Balmer conserve encore son statut de quartier résidentiel en raison de la présence toujours importante de populations riches et d'un habitat de haut standing.

17 Au terme de ces deux phases de littoralisation, se dessine une population hétérogène, où coexistent sans se mélanger riches et pauvres, habitats précaires et logements de haut standing. Mais il convient de nuancer l'association simpliste entre étrangers, statut social élevé et habitat de haut standing d'une part, et autochtones, statut social défavorisé et habitat précaire d'autre part. En effet, la dynamique urbaine portée par une forte attractivité attire aussi des populations autochtones socialement aisées. Parallèlement, d'autres populations étrangères (maliennes, burkinabés et ghanéennes) pratiquant des activités halieutiques (pêcheurs, mareyeurs) finissent également par s'installer à Balmer, participant à complexifier le profil social et géographique de la présence allochtone. Au total, différents types de populations cohabitent sur le littoral.

Tableau 1. Répartition de la population sur le littoral du quartier Balmer.

\begin{tabular}{|c|l|l|c|c|}
\hline $\begin{array}{l}\text { Statut } \\
\text { national }\end{array}$ & $\begin{array}{l}\text { Statut } \\
\text { économique }\end{array}$ & Nationalité/Ethnie & Effectifs & Pourcentage \\
\hline Étranger & \multirow{2}{*}{$\begin{array}{l}\text { Populations } \\
\text { nanties }\end{array}$} & Libanais, Français & 80 & $41 \%$ \\
\cline { 3 - 5 } & $\begin{array}{l}\text { Ivoirien } \\
\text { autres populations locales }\end{array}$ & 43 & $22 \%$ \\
\hline Étranger & $\begin{array}{l}\text { Populations } \\
\text { pauvres }\end{array}$ & $\begin{array}{l}\text { Burkinabé, Ghanéen, } \\
\text { Malien }\end{array}$ & 52 & $27 \%$ \\
\cline { 3 - 5 } & $\begin{array}{l}\text { Dioula, Kroumen, Baoulé, } \\
\text { autres populations locales }\end{array}$ & 19 & $10 \%$ \\
\hline Ivoirien & & 194 & $100 \%$ \\
\hline \multicolumn{2}{|l}{ Total }
\end{tabular}

Source : enquêtes personnelles, E. A. Ahua, janvier 2019

18 Au-delà de leurs statuts socio-économiques distincts, ces différentes populations ont un point commun qui est le développement d'un quotidien lié au littoral. Ces populations pratiquent des activités qui soient se rencontrent, soient s'opposent, entrent en compétition, ou se réinventent et se réagencent dans l'espace (Desse, 2005). Leurs intérêts propres peuvent parfois diverger sur un espace commun où se révèlent de remarquables différenciations et logiques de ségrégation.

\section{Une accessibilité spatiale et socio-économique contrastée du littoral}

La fréquentation du littoral par les différents acteurs en présence s'inscrit dans une logique de différenciation socio-spatiale. Ces dissimilarités entre les populations s'articulent autour de trois éléments principaux que sont l'accessibilité aux services, les équipements balnéaires et les heures de fréquentation. 


\section{Une segmentation de l'espace par l'accès aux services gastronomiques et aux espaces de loisirs}

20 Sur le littoral, il existe plusieurs espaces gastronomiques, de loisirs et buvettes, qui sont les théâtres d'une division. En effet, l'accès aux différents services est lié à la qualité de la prestation et au coût des produits proposés. Concrètement, les populations qui appartiennent à des catégories sociales défavorisées et au niveau de vie faible sont limitées dans leur fréquentation de ces espaces, et sont contraints à la fréquentation de certaines buvettes installées de manière plus ou moins formelle et précaire.

La ségrégation littorale apparaît ici immédiatement visible dans l'accessibilité aux espaces de loisirs et de détente. Les logiques économiques et de fréquentation des sites qui font l'objet d'une segmentation socio-spatiale (restaurants, paillotes, piscines, etc.) sont marquées par une forte sélectivité de la clientèle à travers les coûts élevés de leurs prestations qui excluent préalablement les populations pauvres.

Le caractère aigu du phénomène et son évolution vers davantage de sélectivité nous amène à nous interroger de manière circonstanciée sur la cohabitation entre les différents résidents du littoral et entre les acteurs dont les pratiques de la mer sont différentes (baignade, natation, ou autres activités nautiques comme le surf).

\section{Les flux de fréquentation des plages de Balmer : des temporalités distinctes}

Le quartier de Balmer est l'objet d'une littoralisation croissante et les activités temporaires y sont les plus importantes. Dans de telles circonstances, une dimension nouvelle de la ségrégation trouve son origine dans l'ordre spatio-temporel. Près de la mer, les plus démunis n'ont pas accès aux sites où se trouvent les populations socialement aisées. De même, dans les espaces de baignade, les moments de présence des populations démunies sont marqués par une absence remarquable des populations riches. Une fréquentation de la mer fortement dissociée dans le temps fait du littoral un point d'ancrage spatial de la séparation des acteurs. 
Figure 4. Spatialisation des inégalités sur l'espace littoral du quartier Balmer.

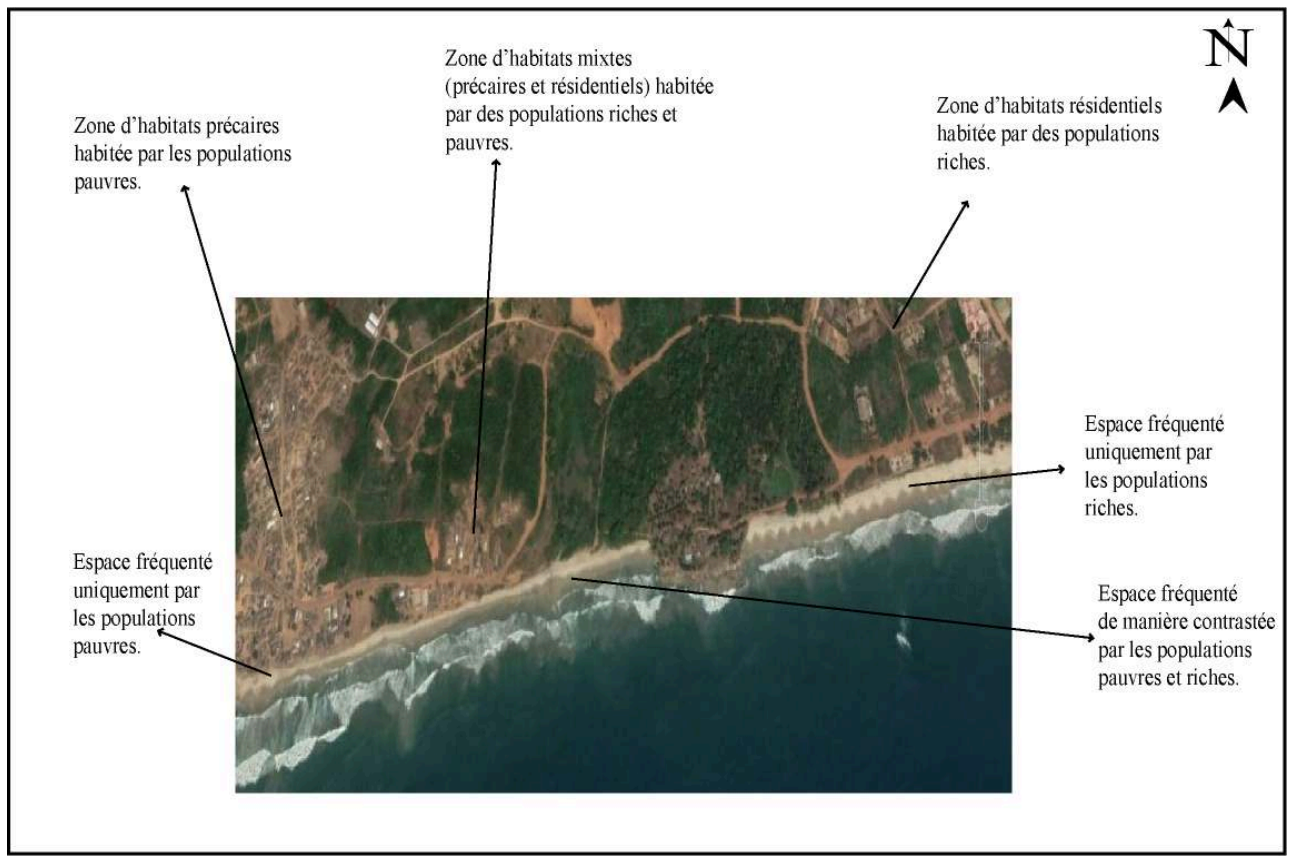

Source: Google Earth, F. Gohourou \& E.A. Ahua, avril 2019

La figure 4 met en exergue les caractéristiques de la ségrégation littorale entre les populations. L'analyse de la spatialisation du phénomène montre que l'espace est divisé en deux zones de partage entre populations riches et pauvres. Les installations balnéaires et les hôtels se sont inscrits dans cette veine : les restaurants et parasols d'un côté, les buvettes spontanées et paillotes de l'autre, etc. En outre, la fréquentation de l'espace obéit à un principe de discontinuité et de fragmentation spatio-temporelle.

Tableau 2. Grille de temporalité des fréquentations du littoral de Balmer.

\begin{tabular}{|c|c|c|c|c|}
\hline & \multicolumn{4}{|c|}{ Statut des populations } \\
\hline & $\begin{array}{l}\text { Population } \\
\text { riche }\end{array}$ & $\begin{array}{l}\text { Population } \\
\text { pauvre }\end{array}$ & $\begin{array}{l}\text { Population } \\
\text { riche }\end{array}$ & $\begin{array}{c}\text { Population } \\
\text { pauvre }\end{array}$ \\
\hline $\begin{array}{l}\text { Période de } \\
\text { fréquentation }\end{array}$ & \multicolumn{2}{|c|}{$\begin{array}{c}\text { Jours ouvrables } \\
\text { (Lundi au Vendredi) }\end{array}$} & \multicolumn{2}{|c|}{$\begin{array}{c}\text { Week-end } \\
\text { (Samedi et Dimanche) }\end{array}$} \\
\hline $\begin{array}{c}\text { Matin } \\
(7 \mathrm{~h}-12 \mathrm{~h})\end{array}$ & & & & \\
\hline $\begin{array}{l}\text { Après-midi } \\
(12 \mathrm{~h}-18 \mathrm{~h})\end{array}$ & & & & \\
\hline $\begin{array}{c}\text { Soir } \\
(18 \mathrm{~h}-21 \mathrm{~h})\end{array}$ & & & & \\
\hline
\end{tabular}

Source : E. A. Ahua, janvier 2019 

davantage un phénomène ségrégatif en pleine expansion où la fréquentation des espaces s'inscrit dans une logique d'affinité et de critères socio-ethniques. Dans le détail, on remarque que les pratiques de week-ends sont plutôt le fait des populations locales et de statut social modeste tandis que les jours ouvrables sont plus l'apanage des populations riches et des touristes. Cette organisation hebdomadaire ponctuée par des rythmes spatio-temporels spécifiques selon le type de population révèle une différenciation sociale. Il arrive cependant que différentes catégories d'acteurs se rencontrent sur certaines plages horaires propices à la co-présence, en particulier les après-midi des jours ouvrables. Il n'est alors pas rare d'observer l'expression de tensions entre ces acteurs, que révèlent des représentations et comportements à caractère hostile et raciste.

\section{La naissance de nouvelles attitudes marquant l'opposition des acteurs littoraux}

Devant un espace de plus en plus identifié comme point de séparation des différents groupes sociaux, les populations vont développer un certain nombre de comportements vis à vis des catégories opposées.

\section{Le développement de la xénophobie et de l'insécurité}

Sur les plages de Balmer, les populations riches ont accès aux restaurants et aux hôtels de luxe et les plus agréables, auxquels les populations socialement modestes n'ont pas accès comme cela a déjà été mentionné.

De même, les populations aisées lors de leurs moments de détente et divertissement près de la mer ont pour habitude de pratiquer des sports nautiques tels que le surf, la voile, la planche à voile, le ski nautique, le jet ski, tandis que les populations pauvres se limitent à la natation et plus généralement à la baignade. Les pratiques contrastées au sein de cet espace somme toute assez réduit apparaissent particulièrement visibles, et renforcent les représentations sociales tranchées et les préjugés réciproques. Les moins nantis, qui se sentent mis à l'écart et fustigés, développent complexes et frustrations. Cette situation semble favoriser le développement de perceptions xénophobes chez certains autochtones de condition modeste, qui parfois se prolongent par des actes racistes, sous la forme d'agressions verbales (injures, menaces, propos diffamatoires, moqueries) ou physiques (vols, dépouillements, braquages). Tant sur les plages qu'au sein des résidences, les spoliations et l'insécurité envers les populations aisées et les touristes sont désormais une réalité quotidienne, et nourrissent une méfiance réciproque entre les acteurs.

\section{Méfiance réciproque, mesures de protection, et nouvelles formes de ségrégation}

Face à l'insécurité croissante, les populations nanties adoptent des mesures de protection et de sécurité. Tant sur les berges qu'au niveau des habitations, celles-ci développent des stratégies de protection contre les populations pauvres. Avec les vols et agressions qui prennent de l'ampleur, les populations riches et étrangères procèdent à une sécurisation de leurs résidences : installation de barbelés le long des murs de leurs habitations, protections murales électriques anti-intrusions activées la nuit, entre 
autres exemples. Les mesures de protection se prolongent lors des sorties sur les plages, les populations aisées étrangères faisant appel à des services de vigiles parfois accompagnés de chiens qu'ils emploient via des contrats avec des sociétés de gardiennage. Ainsi, près de leurs véhicules ou sur les portails des maisons, l'on observe constamment la mention "Attention chien méchant ». Du point de vue de ces populations aisées en situation de vulnérabilité, les dispositions sécuritaires sont des réponses adaptées au comportement hostile de certaines populations pauvres.

\section{Discussion : pratiques contrastées et distances socio- spatiales}

\section{Un phénomène ségrégatif découlant des paramètres environnementaux et de l'existence de pratiques illicites sur les plages}

30 Avec une urbanisation littorale fortement accélérée et mouvementée, la territorialisation des plages et des sites touristiques se fait de plus en plus au gré de personnes de mauvaise foi, en l'occurrence les délinquants, des trafiquants et narcotrafiquants (Desse, 2005). Cette situation exacerbée par l'émergence de pratiques socialement déviantes, de la prostitution et d'agressions multiformes sur la plage de Balmer augmente la vulnérabilité et la perception d'un risque parmi les populations riches et les touristes. En conséquence, celles-ci ont recours à des mesures sécuritaires et de distanciation qui sont en réalité elles-mêmes de nouveaux facteurs de ségrégation. En outre, selon les populations nanties, les populations pauvres ont une gestion de l'espace qui ne respecte pas les normes environnementales avec la création de dépotoirs sauvages et les écoulements abusifs des eaux usées. Cela conforte chez les premières la nécessité perçue de renforcer la séparation avec ces nouveaux acteurs dans un quartier à vocation résidentielle. En effet, les représentations et pratiques adoptées par les populations autochtones pauvres s'inscrivent en contradiction avec l'attractivité initiale de Balmer liée aux caractères ludique et luxueux du lieu incarné par les sites de villégiature faisant l'objet de perceptions positives (piscines, hôtels, parcs d'embellissement, espaces gastronomiques, etc.). Cependant, la fréquentation contrastée de cet espace est analysée au sens large par Devienne (2015) comme le résultat de la pression collective sur ces espaces résidentiels. Elle poursuit en remarquant que ces pressions engendrent par leurs conséquences des ségrégations de type formel ou informel sur un espace littoral fortement urbanisé. En confortant la coprésence de populations socialement diverses sur des espaces limités, l'urbanisation produit de la ségrégation (figure 3), tant dans les modes d'habiter que dans les pratiques quotidiennes et la construction de perceptions sociales et spatiales en lien avec ce quotidien.

\section{L'influence de l'urbanisation dans l'évolution de la ségrégation littorale}

31 La ségrégation littorale à San-Pédro s'accompagne d'une mise à distance des acteurs, dans un espace polarisé et segmenté par deux catégories de population. Un phénomène que la poussée démographique et l'extension urbaine ne font que conforter avec le 
temps. Pour Pouyane (2006), l'étalement urbain et la ségrégation socio-spatiale sont deux tendances majeures de l'urbanisation contemporaine. Selon l'auteur, la redistribution du peuplement accompagnant l'expansion de l'espace urbain renforce la polarisation spatiale des groupes sociaux différenciés selon le statut économique, l'origine sociale, et les motivations de leur installation. Pendall et Carruther (2003) précisent que l'étalement urbain n'engendre pas systématiquement de la ségrégation, mais influence plutôt les facteurs et les mécanismes à l'origine de ce phénomène (figure 5).

Figure 5. Etalement urbain et processus ségrégatif.

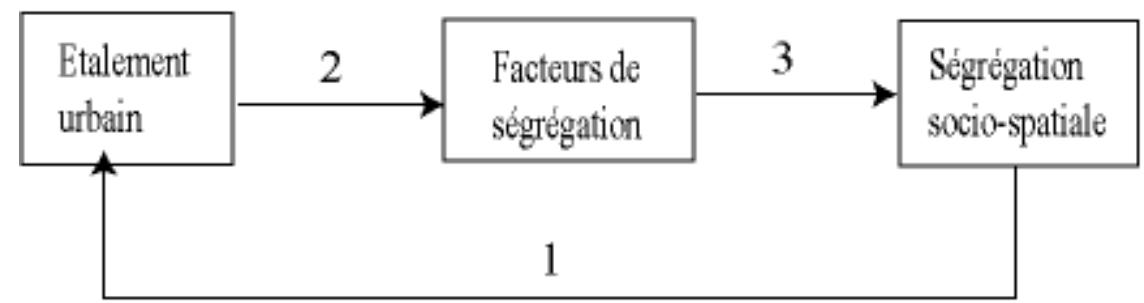

Source : Pendall et Carruther (2003)

L'étalement urbain contribue notamment à renforcer la ségrégation via la flambée du foncier (Dabet et Flosch, 2014), ce que nous constatons à San-Pédro. Ainsi, nos enquêtes ont révélé que l'arrivée de populations aisées s'est accompagnée d'une inflation remarquable du coût du foncier, notamment sur le littoral. La valeur des terrains est passée d'une fourchette de 1,5-2,8 millions de francs CFA en 2000 à 3-5 millions de francs CFA en 2008, puis à 4-8 millions de francs CFA en 2012. La valeur d'achat des résidences dans la zone a suivi une tendance analogue, en s'appréciant de 3,5-7,5 millions de FCFA en 2000 à 9-18 millions de FCFA en 2008, puis à 13,5-25 millions de FCFA. ${ }^{1}$ L'évolution des loyers mensuels des logements les plus modestes s'est avérée plus raisonnable. Elle a néanmoins enregistré un doublement au cours de la période, passant de 200000 à $400000 \mathrm{FCFA}$, un niveau rédhibitoire pour les ménages locaux qui habitaient traditionnellement la zone. Cela induit une pluralité de processus discriminatoires et ségrégatifs, tangibles dans le cas de San-Pédro où les catégories aisées (grands commerçants, industriels, etc.) majoritairement étrangères sont préférentiellement localisées sur le littoral de Balmer, à bonne distance des populations pauvres constituées d'autochtones et allochtones des pays voisins.

\section{Ségrégation socio-spatiale, conflits d'usages et absence de facto de la puissance publique}

33 Sur le littoral, dans les zones faisant l'objet d'une forte pression foncière et de pratiques concurrentes et différenciées de l'espace, de multiples formes de ségrégation se conjuguent et génèrent des tensions sociales. Par exemple, les conceptions différentes de la nudité et de ce qui est acceptable ou pas dans l'espace public (harcèlement de touristes arborant leurs seins nus sur la plage, etc.) illustre bien la façon dont des représentations divergentes selon les acteurs peuvent alimenter la différenciation sociale et la segmentation des pratiques et de l'appropriation de l'espace.

La forte prééminence de la loi du marché dans les logiques observées sur le littoral de Balmer révèle aussi en creux l'absence des pouvoirs publics et de la mise en place de 
politiques pour accompagner les mutations rapides du territoire urbain. L'importance des enjeux urbains et des conflits sociaux que cristallise cet espace rend pourtant impérieuse la mise en œuvre d'une forme de régulation de la puissance publique pour assurer la pérennité du vivre ensemble. Ici comme dans d'autres espaces littoraux, les politiques ont pendant longtemps négligé la question de la ségrégation, se focalisant uniquement sur la ségrégation urbaine dans les domaines du logement, des transports, de l'école, de l'accès aux services et équipements (Devienne, 2016). Pour Fusco et Scarella (2011), cela est en partie dû au développement d'une appréhension avant tout quantitative de la ségrégation par le politique, faisant passer au second plan les considérations qualitatives (perceptions et pratiques des habitants et usagers) pourtant bien présentes sur le littoral, comme le montre le cas de Balmer. Une telle contradiction appelle à la prise en compte d'approches novatrices susceptibles d'aider à comprendre ce phénomène et à y trouver des solutions, dans une société ivoirienne en forte croissance démographique et où les littoraux concentrent une part croissante de la population, et une diversité toujours plus importante d'acteurs.

\section{Conclusion}

Au terme de cette étude, il apparaît que le littoral de Balmer a connu deux grandes phases de littoralisation impulsées par l'essor du tourisme et les mobilités résidentielles ainsi que par le dynamisme des activités économiques halieutiques et informelles. Les différents acteurs en présence ont développé une multiplicité de territorialités liées à la pratique de la plage et de ses environs, répondant à une diversité de statuts socio-économiques et de référents culturels. L'espace littoral enjeu de ces pratiques et représentations a alors été le théâtre de logiques de morcellement et de bipolarisation entre population riche et population pauvre. Les logiques concernées ont notamment trait aux disparités dans l'accessibilité aux équipements balnéaires et aux fréquentations discontinues de la plage dans le continuum spatiotemporel. La ségrégation devient alors une véritable préoccupation en raison de l'apparition de pratiques de distanciation et de mœurs nouveaux, et de perceptions xénophobes et racistes qu'elle suscite auprès des populations. Ces perceptions ellesmêmes contribuent à alimenter à leur tour la distanciation selon le statut social et l'origine. Enfin, l'insécurité grandissante à Balmer participe à changer la perception de la zone et à discréditer ce secteur qui avait construit son attractivité sur l'image d'un espace paisible, calme, et où il faisait bon vivre. Les facteurs de la ségrégation littorale sont indissociables de l'urbanisation galopante de la ville de San-Pédro. Les politiques publiques d'aménagement du littoral urbain doivent faire de ce nouveau défi une priorité, sous peine de voir la cohésion sociale et la perception du quartier et de la ville, l'une des plus importantes du pays, se dégrader. 


\section{BIBLIOGRAPHIE}

AUDREN G., BABY-COLLIN V. (2017), « Ségrégation socio-spatiale et ethnicisation des territoires scolaires à Marseille ", Belgeo, 2-3.

BIGNOUMBA G.S. (2000), « Anthropisation et conflits d'usages sur le littoral du Gabon : Éléments de réflexion », Cahier nantais, 53, pp. 107-114.

CADORET A. (2006), Conflits d'usages liés à l'environnement et réseaux sociaux : Enjeux d'une gestion intégrée ? Le cas du littoral de Languedoc-Roussillon, Thèse de doctorat, Université de Montpellier III - Paul Valery, $591 \mathrm{p}$.

DABET G., FLOSCH J.M. (2014), La ségrégation spatiale dans les grandes unités urbaines de France métropolitaine : Une approche par les revenus, Coll. Documents de travail, Insee, 43 p.

DESSE M. (2005), « Territorialisation et enclaves littorales dans les îles de la Caraïbe », Études caribéennes, 3, pp. 9-14.

DEVIENNE E. (2015), « Controverse à Los Angeles : Le port du maillot de bain en ville du XX siècle », Revue d'histoire du vêtement et de la mode, Université de Lille, pp. 174-193.

DEVIENNE E. (2016), « La question raciale sur le littoral de Los Angeles (années 1920-1980) », Les gauches et les colonies, Fondation Nationale des Sciences politiques, pp. $109-124$.

FUSCO G., SCARELLA F. (2011), « Métropolisation et ségrégation socio-spatiale : Les flux de mobilités résidentielles géographiques en Provences Alpes Côtes d'Azur », L'Espace Géographique, 40, pp. 319-336.

GOHOUROU F. (2012), « Territorialité française dans l'agglomération abidjanaise : l'exemple de la commune de Marcory », Actes de colloque Tome 1 «Les perspectives de la Géographie en Afrique Subsaharienne », Sarrebruck, éd. EUE, pp. 249-263.

GUYOT S. (2009), L'environnement contesté : La territorialisation des conflits environnementaux sur le littoral du Kwazulu Natal (Afrique du Sud : Kosi bay, St Lucia, Richars Bay et Port Shepstone), Thèse de doctorat, Université Paris X - Nanterre, $510 \mathrm{p}$.

INSTITUT NATIONAL DE LA STATISTIQUE (1975), Recensement général de la population et de l'habitat (RGPH).

INSTITUT NATIONAL DE LA STATISTIQUE (1988), Recensement général de la population et de l'habitat (RGPH).

INSTITUT NATIONAL DE LA STATISTIQUE (1998), Recensement général de la population et de l'habitat (RGPH).

INSTITUT NATIONAL DE LA STATISTIQUE (2014), Recensement général de la population et de l'habitat (RGPH).

PENDALL R., CARRUTHERS J. (2003), “Does density exacerbate income segregation? Evidence from U.S. metropolitan areas, 1980 to 2000”, Housing Policy Debate, 14, 4, pp. 541-589.

POGNONEC A. (2016), « Le littoral : Cap sur les aspects juridiques », Études marines, Centre d'étude stratégique de la marine, pp. 28-40.

POUYANE G. (2006), « Étalement urbain et ségrégation socio-spatiale : Une revue de littérature », Cahier du GRES, 7, pp. 1-13. 
PRETECEILLE E. (2009), « La ségrégation ethno-raciale a-t-elle augmenté dans la métropole parisienne? ", Revue française de sociologie, 50, pp. 489-819.

ROBERT S. (2008), La vue sur mer et l'urbanisation du littoral. Approche géographique et cartographique sur la Côte d'Azur et la Riviera du Ponant, Thèse de doctorat, Université de Nice Sophia-Antipolis, $459 \mathrm{p}$.

SECK A. (2014), Les pêcheurs migrants de Guet-Ndar (Saint-Louis du Sénégal) : Analyse d'une territorialité diverse entre espaces de conflits et espaces de gestion, Thèse de doctorat, Université de Liège et Université Cheick Anta Diop, 356 p.

\section{NOTES}

1. Source : E. A. Ahua, enquête de terrain, 2019.

\section{RÉSUMÉS}

Depuis plusieurs décennies, la littoralisation des côtes est un phénomène qui prend de l'importance dans un contexte de croissance démographique exponentielle et de forte expansion de l'espace bâti. Dans les pays développés comme dans les pays en développement, ce phénomène de littoralisation à différentes échelles s'accompagne d'un certain nombre de disparités sociales et environnementales, qui se traduisent par une ségrégation socio-spatiale. Ainsi, l'étude des rapports entre littoralisation et ségrégation se pose avec intérêt dans le champ de la géographie. Dans le quartier Balmer à San-Pédro, ville située au sud-ouest de la Côte d'Ivoire, les processus de ségrégation et de différenciation sociale sont de plus en plus récurrents sur le littoral. Dans ce cadre, la présente contribution dresse un état des lieux des différentes modalités de littoralisation et s'intéresse aux différents réseaux d'acteurs littoraux ainsi qu'aux facteurs qui sont à l'origine de la ségrégation socio-spatiale dans le secteur littoral de Balmer. S'appuyant sur une revue de littérature, une enquête par questionnaire, des entretiens semi-directifs et une observation directe, les principaux résultats de l'étude indiquent que la littoralisation de Balmer a été induite par une économie résidentielle, touristique et un dynamisme des activités industrielles, halieutiques et informelles. En outre, il ressort de cette réflexion que cette littoralisation est l'apanage d'une population hétérogène (riche et pauvre, locale et étrangère) qui se livre une compétition pour l'accès à la plage, aux services gastronomiques et de loisirs. Il en résulte une opposition spatio-temporelle dans la fréquentation de ces espaces par ces différents groupes sociaux. Cette situation se traduit par une segmentation de l'espace littoral et la naissance de perceptions et comportements xénophobes voire racistes.

For several decades, coastal littoralisation has been a phenomenon of growing importance in a context of exponential demographic growth and strong expansion of the built space. In both developed and developing countries, this phenomenon of littoralisation at different scales is accompanied by social and environmental disparities, which are reflected in socio-spatial segregation. Thus, the study of the relationships between littoralisation and segregation appears to be more and more an issue of interest in the field of geography. In the Balmer district of SanPédro, a town in the southwest of Ivory Coast, the processes of segregation and social 
differentiation are more and more recurrent on the coast. In this context, this contribution draws up an inventory of the different modes of coastalisation and focuses on the different networks of coastal actors as well as on the explanations that account for socio-spatial segregation in the coastal sector of Balmer. Based on a literature review, a questionnaire survey, semi-structured interviews and direct observation, the main results of the study indicate that Balmer's coastal development was induced by a residential and tourist economy and the dynamics of industrial, fishery and informal activities. In addition, it points out the fact that coastalisation is the prerogative of a heterogeneous population (rich and poor, local and foreign) whose various segments are competing for access to the beach, restaurants and leisure services. The result is a spatio-temporal opposition in the use of space by the different social groups. This situation results in a segmentation of the coastline and the rise of xenophobic and even racist perceptions and behavior.

\section{INDEX}

Keywords : littoral, littoralisation, social segregation, spatial segregation, Balmer, San-Pédro, Ivory Coast

Mots-clés : littoral, littoralisation, ségrégation sociale, ségrégation spatiale, Balmer, San-Pédro, Côte d'Ivoire

\section{AUTEURS}

\section{FLORENT GOHOUROU}

Géographe, Maître-assistant, Université Jean Lorougnon Guédé (Daloa - Côte d'Ivoire), Chercheur associé - MIGRINTER (UMR 7301, CNRS - Université de Poitiers), fgohourou@yahoo.com

\section{CÉDRIC AUDEBERT}

Géographe, Directeur de recherche au CNRS, Laboratoire Caribéen de Sciences Sociales, UMR 8053, CNRS (Université des Antilles), cedric.audebert@cnrs.fr

\section{ÉMILE AURÉLIEN AHUA}

Université F.H.B (Abidjan - Côte d'Ivoire), Université de Nantes (LEGT - UMR 6554), emailoaurelien@gmail.com 